\title{
Rescattering in meson photoproduction from few body systems
}

\author{
J.-M. Laget \\ Thomas Jefferson National Accelerator Facility, Newport News, Virginia 23606, USA
}

(Received 14 July 2005; published 10 April 2006)

\begin{abstract}
Exclusive reactions induced at high momentum transfer in few body systems provide us with an original way to study the production and propagation of hadrons in cold nuclear matter. In very well-defined parts of the phase space, the reaction amplitude develops a logarithmic singularity. It is on solid ground since it depends on only on-shell elementary amplitudes and on low momentum components of the nuclear wave function. This is the best window for studying the propagation of exotic configurations of hadrons such as the onset of color transparency. It may appear earlier in meson-photoproduction reactions, more particularly in the strange sector, than in the more classical quasi-elastic scattering of electrons. More generally, those reactions provide us with the best tool to determine the cross section of the scattering of various hadrons (strange particles, vector mesons) from the nucleon and to obtain the production of possible exotic states.
\end{abstract}

DOI: 10.1103/PhysRevC.73.044003

PACS number(s): 13.60.Le, 12.40.Nn, 25.10.+s

\section{INTRODUCTION}

Exclusive reactions induced at high momentum transfer in few body systems provide us with an original way to study the production and propagation of hadrons in cold nuclear matter. In very well-defined parts of the phase space, the reaction amplitude develops a logarithmic singularity that enhances the cross section. Here, the reaction amplitude is on solid ground since it depends on only on-shell elementary amplitudes and on low momentum components of the nuclear wave function [1]. On the one hand this allows the determination of the scattering cross section of short-lived particles. On the other hand this allows the study of the propagation of exotic configurations such as the onset of color transparency.

The concept of color transparency follows from the underlying structure of QCD: Interactions between "white" objects depend on their transverse size [2,3]. A hard scattering of the probe produces recoiling particles with small transverse size whose subsequent interactions in nuclear matter are reduced. There is no doubt that color transparency should occur. The question is where and when.

The difficulty resides in the fact that such an exotic configuration evolves quickly toward the asymptotic state of the detected hadron: To observe color transparency, the characteristic scale of this evolution should be larger than, or comparable with, the size of the largest nuclei.

To date there is no convincing evidence for color transparency in photon- and electron-induced reactions. The reason is that most of the attempts were performed in semiexclusive kinematics. In the $A\left(e, e^{\prime} p\right)$ reactions [4,5] for instance, it is very likely that the values of the only available hard scale, $Q^{2}$, are too low to observe color transparency in the quasifree kinematics channels, where the energy of the ejected nucleon $T_{p}$ and the photon four-momentum are not independent $\left(T_{p}=\right.$ $\left.Q^{2} / 2 M\right)$. In the range of values of $Q^{2}$ accessible today, the lifetime of the small object is of the order of the distance between nucleons rather than the nuclear radius. For instance, at the highest $Q^{2}=6 \mathrm{GeV}^{2}$ where data exist, the energy of the outgoing proton is only $3 \mathrm{GeV}$ and its characteristic evolution distance [6] is no more than $1.5 \mathrm{fm}$, closer to the internucleonic distance rather than the size of the nucleus.

A signal has been reported in $A\left(e, e^{\prime} \rho\right)$ reactions, at Fermi Lab. [7] and DESY [8]. However, it comes from a subtle interplay between the attenuation of the hadronic component of the virtual photon in the entrance channel and the onset of color transparency in the exit channel (see, e.g., Ref. [9]). An experiment [10] has been completed recently at Jefferson Laboratory (JLab) to disentangle these two effects: One has to await the final analysis for a more definite answer.

The way to overcome these difficulties is to study reactions induced by photons in few body systems: Exclusive reactions allow the formation length of the hadron to be adjusted to the distance between nucleons [11-13]. The kinematics should be chosen so that the interactions of the emerging hadron with a second nucleon are maximal. This occurs when the produced hadron propagates on-shell and rescatters on a second nucleon at rest (triangular logarithmic singularity). A clear signal for color transparency would be the suppression of the final-state interaction peak when the momentum transfer increases. This situation is more comfortable than in the more classical study of quasi-elastic scattering of electrons from heavy nuclei, in which one looks for a change of a flat level of attenuation of the outgoing nucleon instead of the evolution of a well-defined peak.

This conjecture [11-13] has been tested in two studies of the ${ }^{2} \mathrm{H}\left(e, e^{\prime} p\right) n$ reaction that were completed recently at JLab: The first [14] with two magnetic spectrometers in Hall A; the second [15] with the CEBAF Large Acceptance Spectrometer [16] (CLAS) in Hall B. The preliminary results do not exhibit a signal of color transparency (within the experimental and theoretical uncertainties) in the range $2<Q^{2}<6 \mathrm{GeV}^{2}$ when compared with the latest prediction [17] of the various interaction mechanisms.

It may occur earlier in the exclusive photoproduction of mesons. The reason is that mesons are made up of two quarks. They may recombine more easily through the exchange of one hard gluon only and prepare a configuration with a small transverse extension that further evolves toward its 
asymptotic size. The second reason is that the hard scale is provided by the four-momentum transfer $t$ that happens to define the interaction volume, while the virtuality $Q^{2}$ of the photon that is exchanged in $\left(e, e^{\prime} p\right)$ reactions defines the scale of observation [18].

Indeed, a hint was reported in the ${ }^{4} \mathrm{He}\left(\gamma, p \pi^{-}\right)$and ${ }^{16} \mathrm{O}\left(\gamma, p \pi^{-}\right)$channels recently studied at JLab $[19,20]$. However, the signal is weak and again these are semiexclusive reactions. The signal must be confirmed by completely exclusive measurements.

The simplest example is the reaction ${ }^{2} \mathrm{H}\left(\gamma, p \pi^{-}\right) p$ in the energy range $4<E_{\gamma}<10 \mathrm{GeV}$. For real photons the momentum transfer $t$, between the incoming photon and the outgoing pion, sets the size of the interaction volume. As can be seen in Fig. 1, the on-shell rescattering peaks corresponding to $\pi p$ or $p p$ interactions are clearly separated. Such a logarithmic singularity has already been observed at lower energies [21]. At the top of each peak, the rescattering amplitude is dominated by low momentum components of the deuteron wave function and on-mass-shell elementary reaction amplitudes (see Ref. [1]). The elementary reaction $n\left(\gamma, \pi^{-}\right) p$ is well reproduced by a model based on the exchange of saturating Regge trajectories [22]. The $\pi$-nucleon, as well as the nucleon-nucleon, elementary scattering amplitudes are almost entirely absorptive and well under control at high energy [23,24]. The rescattering

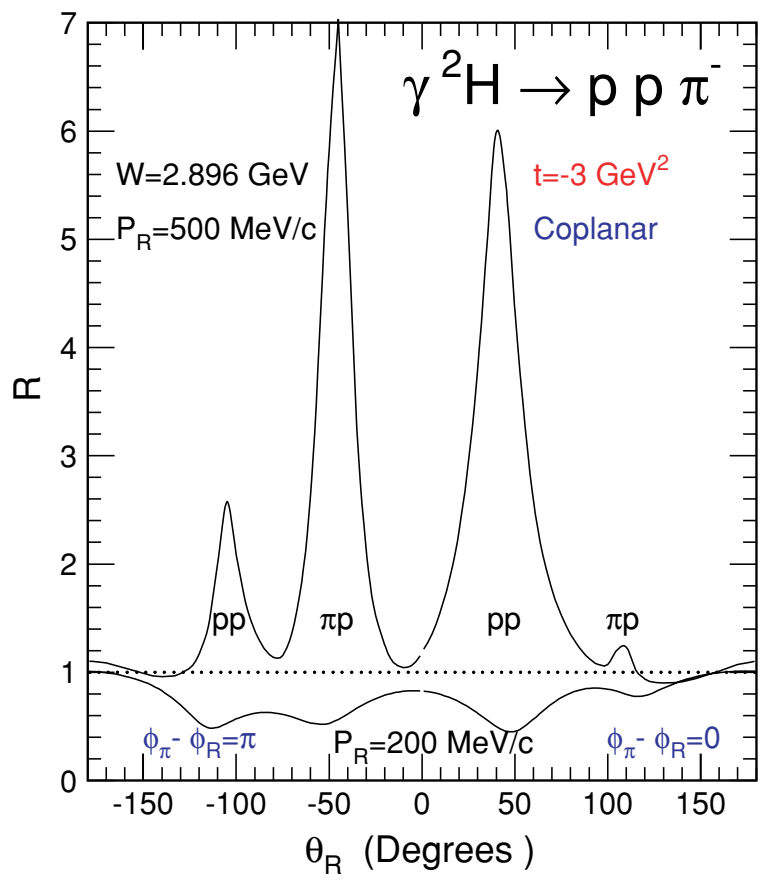

FIG. 1. (Color online) Ratio of the total to the quasi-free cross section of the ${ }^{2} \mathrm{H}\left(\gamma, \pi^{-} p\right) p$ reaction against the angle of the recoiling proton whose momentum is kept constant at $500 \mathrm{MeV} / c$ (top) and $200 \mathrm{MeV} / c$ (bottom). The peaks labeled $\pi p$ and $p p$ correspond, respectively, to $\pi p$ and $p p$ on-shell rescattering. The dotted line corresponds to the quasi-free process. The kinematics is coplanar, and positive angles correspond to the emission of the pion and the recoiling proton on the same side of the photon. amplitudes are therefore on solid ground [17]: The method can be calibrated at low values of the four-momentum transfer $t$. A signal of color transparency would be the reduction of the $\pi p$ rescattering peak when $t$ increases. It could happen sooner in the strange sector, where the strange quark may play a special role.

Alternatively, the method will allow a determination of the cross section of hyperons $(\Lambda \ldots)$ or vector-meson $(\phi, J / \Psi \ldots)$ scattering with nucleons, or the interactions between other unstable hadrons. More generally it offers us a way to obtain the mechanisms of the formation of hadrons in cold nuclear matter.

I have already presented these conjectures in several conference talks $[11,25,26]$ and prospect reports. In the meantime, the CLAS collaboration at JLab has completed, with unprecedented statistics, a study [27] of the interactions of a real photon beam (maximum energy $3.7 \mathrm{GeV}$ ) on a deuterium target: It provides us with a unique testing ground of these ideas and the method.

This paper is not one more attempt to predict the size of the signal of color transparency: Too many parameters are unknown, and, although we already know what the qualitative expectations are, only experiments will allow us to quantify the effect. This paper is rather an attempt to provide a comprehensive recollection and update of the various matrix elements in the meson-production sectors and to provide a baseline calculation in a dedicated kinematical range, already accessible at JLab at $6 \mathrm{GeV}$ and its further upgrade to $12 \mathrm{GeV}$, from which any deviation will be meaningful. The nucleon sector was already revisited in Ref. [17]. Section II deals with the pion-production sector, Sec. III deals with the single-kaon-production sector, Sec. IV deals with the vector-meson-production sector (more specifically $\phi$ and $J / \Psi)$, Sec. V addresses issues in color transparency, Sec. VI investigates the possible implication of the search of exotics, and Sec. VII concludes and summarizes the prospects.

\section{THE ${ }^{2} \mathrm{H}\left(\gamma, \pi^{-} p\right) p$ REACTION}

\section{A. The model}

The model is a straightforward update of the diagrammatic approach [1] that has been successful in the analysis of meson-production reactions at lower energies (let's say in the resonance region). It is particularly well suited for evaluating the reaction amplitude near the singularities of the $S$ matrix. The kinematics, the elementary operators as well as the propagators, is relativistic. The deuteron wave function corresponds to the Paris potential [28], but any modern wave function leads to very similar results in the momentum range covered by this study.

Let $k=(v, \vec{k}), p_{D}=\left(M_{D}, \overrightarrow{0}\right), p_{\pi}=\left(E_{\pi}, \vec{p}_{\pi}\right), p_{1}=\left(E_{1}\right.$, $\left.\vec{p}_{1}\right)$, and $p_{2}=\left(E_{2}, \vec{p}_{2}\right)$ be the four-momenta, in the lab system, of, respectively, the incoming photon, the target deuteron, the outgoing pion, the slow outgoing proton, and the fast outgoing proton. The five-fold fully differential cross section is related to the square of the coherent sum of the matrix elements as 
follows:

$$
\begin{aligned}
\frac{d \sigma}{d \vec{p}_{1}\left[d \Omega_{\pi}\right]_{\mathrm{c} . \mathrm{m} .2}}= & \frac{1}{(2 \pi)^{5}} \frac{\left|\vec{\mu}_{\mathrm{c} . \mathrm{m} .}\right| m^{2}}{24|\vec{k}| E_{1} Q_{f}} \\
& \times \sum_{\epsilon, M, m_{1}, m_{2}} \mid \sum_{i=\mathrm{I}}^{\mathrm{III}} \mathcal{M}_{i}\left(\vec{k}, \epsilon, M, \vec{p}_{\pi}, \vec{p}_{1}, m_{1},\right. \\
& \left.\vec{p}_{2}, m_{2}\right)-\mathcal{M}_{i}\left(\vec{k}, \epsilon, M, \vec{p}_{\pi}, \vec{p}_{2},\right. \\
& \left.m_{2}, \vec{p}_{1}, m_{1}\right)\left.\right|^{2}
\end{aligned}
$$

where $\epsilon$ is the polarization vector of the photon and $M, m_{1}$, and $m_{2}$ are the magnetic quantum numbers of the target deuteron and the two outgoing protons, respectively. The norm of the spinors is $\bar{u} u=1$. The amplitudes are computed in the lab frame. The antisymmetry between the two outgoing protons is ensured by the exchange of the role of $\left(\vec{p}_{1}, m_{1}\right)$ and $\left(\vec{p}_{2}, m_{2}\right)$ in the second amplitude. The cross section is differential in the lab three-momentum of proton 1 , but in the solid angle of the pion expressed in the center-of-mass (c.m.) frame of the pair made by the pion and proton 2 . In this frame, the momentum of the pion is $\vec{\mu}_{\text {c.m. }}$ and the total energy is $Q_{f}=$ $\sqrt{\left(E_{\pi}+E_{2}\right)^{2}-\left(\vec{p}_{\pi}+\vec{p}_{2}\right)^{2}}$.

The cross section and the amplitudes are given for the case of a real photon-induced reaction that I consider in this study. They depend on only the transverse components $J_{X}$ and $J_{Y}$ of the hadronic current $\vec{J}$. In the case of a virtual photon beam, additional terms in the cross section are related, as outlined in Ref. [29], to the longitudinal component of the hadronic current $J_{z}$. The matrix elements are expressed as the scalar product $\vec{J} \cdot \vec{\epsilon}$ from which each component of the hadronic

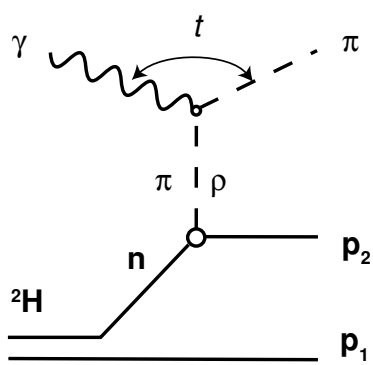

(I)

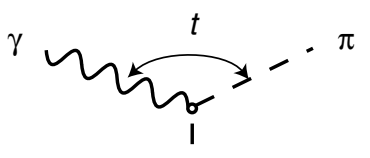

$\pi$ । $\rho$

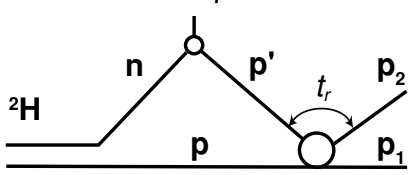

(III)

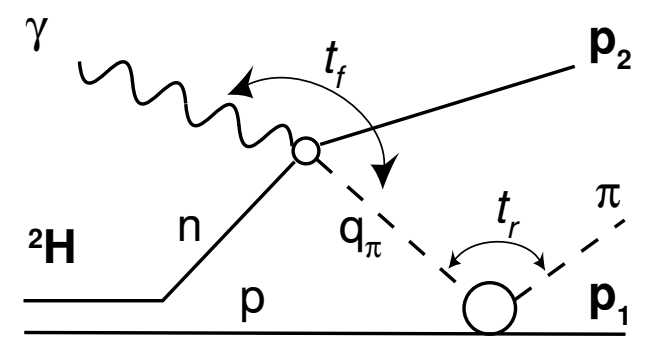

(II)
FIG. 2. The relevant mechanisms: (I) quasi-free, (II) mesonnucleon rescattering, (III) nucleon-nucleon rescattering. current can be deduced. In the following discussion I give the expressions of the dominant reaction amplitudes in Fig. 2 and discuss their update relevant to the high-energy domain accessible at JLab. I refer the reader to [30] for more technical details.

\section{Quasi-free meson production}

The matrix element of the quasi-free amplitude [graph (I) in Fig. 2] takes this simple form:

$$
\begin{aligned}
& \mathcal{M}_{\mathrm{I}}\left(\vec{k}, \epsilon, M, \vec{p}_{\pi}, \vec{p}_{1}, m_{1}, \vec{p}_{2}, m_{2}\right) \\
& =i \sum_{m_{n} m_{l} m_{s}} \sum_{l s}\left(l m_{l} s m_{s} \mid 1 M\right)\left(\frac{1}{2} m_{n} \frac{1}{2} m_{1} \mid s m_{s}\right) \\
& \quad \times u_{l}\left(\left|\vec{p}_{1}\right|\right) Y_{l}^{m_{l}}\left(\vec{p}_{1}\right) T_{\gamma n}\left(\vec{p}_{2}, m_{2},-\vec{p}_{1}, m_{n}\right),
\end{aligned}
$$

where $u_{0}$ and $u_{2}$ are the $S$ and $D$ components of the deuteron Paris wave function [28] and $T_{\gamma n}$ is the amplitude of the elementary $n\left(\gamma, \pi^{-}\right) p$ reaction. I use the on-shell expression (see appendix) of the Regge amplitude of Ref. [22], which is based on the exchange of the saturating Regge trajectories of the pion and the $\rho$ mesons. It leads to a good description of the differential cross section of the $p\left(\gamma, \pi^{+}\right) n$ reaction at large momentum transfer $-t$ in the photon energy range of JLab (around $4 \mathrm{GeV}$ ). As shown in Fig. 3, it leads also to a fair accounting of the more recent JLab data [31] in the $\pi^{-}$channel. I refer the reader to [22] for a thorough presentation of this Regge model and the choice of the coupling constants and parameters: I use the same in this study, except for the cutoff

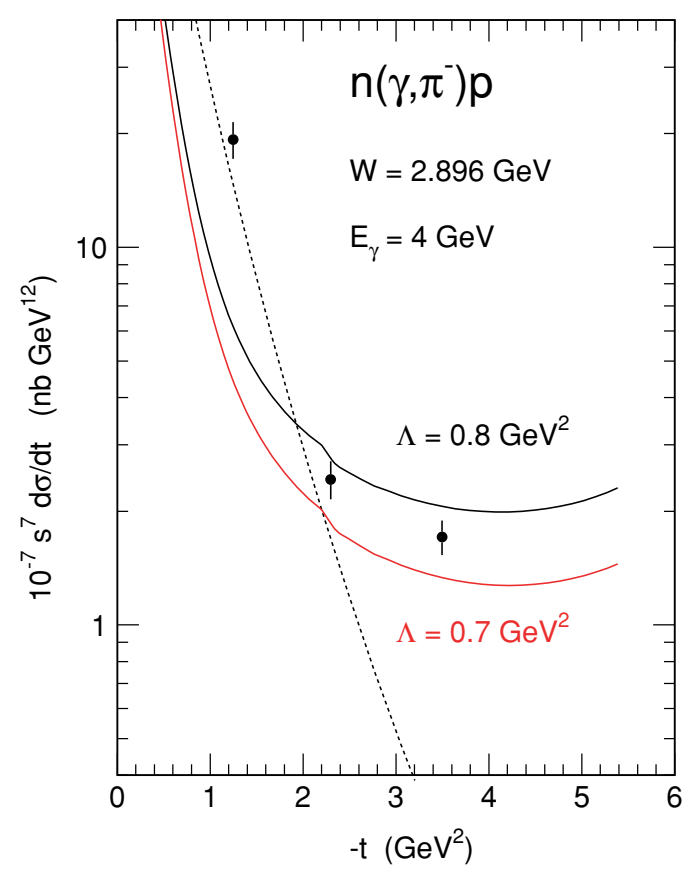

FIG. 3. (Color online) The cross section of the elementary reaction $n\left(\gamma, \pi^{-}\right) p$ at $E_{\gamma}=4 \mathrm{GeV}$. The curves are the prediction of the Regge model. The dashed curve corresponds to linear trajectories. The solid curves correspond to saturating trajectories for two choices of the cutoff mass in the hadronic form factor. The data have been recently recorded at JLab [31]. 
mass of the hadronic form factor, which I chose to be $\Lambda=0.7$ $\mathrm{GeV}^{2}$ instead of $0.8 \mathrm{GeV}^{2}$ in Ref. [22].

When the momentum $\vec{p}_{1}$ of one of the protons is low, only one amplitude dominates cross section (1), which takes this simple form $[1,30]$ :

$$
\frac{d \sigma}{d \vec{p}_{1} d \Omega_{\pi}}=\left(1+\beta_{1} \cos \theta_{1}\right) \rho\left(\left|\vec{p}_{1}\right|\right) \frac{d \sigma}{d \Omega_{\pi}}\left(\gamma n \rightarrow \pi^{-} p\right),
$$

where $\beta_{1}=p_{1} / E_{1}$ and $\theta_{1}$ are the velocity and the angle of the spectator nucleon, respectively. This is nothing but the relation between the yield and the elementary cross section of the production of a pion on a nucleon that moves with the velocity $-\vec{\beta}_{1}$. The number of target nucleons is $\rho\left(\left|\vec{p}_{1}\right|\right) d \vec{p}_{1}$, where $\rho\left(\left|\vec{p}_{1}\right|\right)$ is the momentum distribution of the neutron in deuterium, while $\left(1+\beta_{1} \cos \theta_{1}\right)$ is the flux of photons seen by the moving target nucleon.

\section{Meson-nucleon rescattering}

The matrix element of the pion-proton rescattering amplitude [graph (II) in Fig. 2] takes this form:

$$
\begin{aligned}
\mathcal{M}_{\mathrm{II}} & \left.\vec{k}, \epsilon, M, \vec{p}_{\pi}, \vec{p}_{1}, m_{1}, \vec{p}_{2}, m_{2}\right) \\
= & i \sum_{m_{n} m_{p}}\left(\frac{1}{2} m_{n} \frac{1}{2} m_{p} \mid 1 M\right) \int \frac{d^{3} \vec{p}}{(2 \pi)^{3}} \frac{u_{0}(p)}{\sqrt{4 \pi}} \frac{1}{q_{\pi}^{2}-m_{\pi}^{2}+i \epsilon} \\
& \times \frac{m}{E_{p}} T_{\gamma n}\left(\vec{p}_{2}, m_{2},-\vec{p}, m_{n}\right) T_{\pi N}\left(\vec{p}_{1}, m_{1}, \vec{p}, m_{p}\right) \\
& +D \text { wave part. }
\end{aligned}
$$

The integral runs on the three-momentum of the spectator proton in the loop, which has been put on-shell, $p^{0}=E_{p}=$ $\sqrt{\vec{p}^{2}+m^{2}}$, by the integration over its energy $p^{0}$. It can be split into two parts:

$$
\mathcal{M}_{\mathrm{II}}=\mathcal{M}_{\mathrm{II}}^{\mathrm{on}}+\mathcal{M}_{\mathrm{II}}^{\mathrm{off}}
$$

The singular part of the rescattering integral runs between the minimum and the maximum values of the momentum of the spectator proton in the loop for which the pion can propagate on-shell:

$$
\begin{aligned}
& p_{\min }(p \pi)=\frac{P}{Q_{s}} E_{\text {c.m. }}-\frac{E}{Q_{s}} p_{\text {c.m. }}, \\
& p_{\max }(p \pi)=\frac{P}{Q_{s}} E_{\text {c.m. }}+\frac{E}{Q_{s}} p_{\text {c.m. }},
\end{aligned}
$$

where $E=E_{\pi}+E_{1}, \vec{P}=\vec{p}_{\pi}+\vec{p}_{1}$, and $Q_{s}=\sqrt{E^{2}-\vec{P}^{2}}$ are, respectively, the energy, the momentum, and the mass of the scattering $\pi p$ pair. The momentum and energy of the spectator proton in the c.m. frame of the $\pi p$ pair are

$$
\begin{aligned}
& p_{\text {c.m. }}=\frac{\sqrt{\left[Q_{s}^{2}-\left(m+m_{\pi}\right)^{2}\right]\left[Q_{s}^{2}-\left(m-m_{\pi}\right)^{2}\right]}}{2 Q_{s}}, \\
& E_{\text {c.m. }}=\sqrt{p_{\text {c.m. }}^{2}+m^{2}}=\frac{Q_{s}^{2}+m^{2}-m_{\pi}^{2}}{2 Q_{s}} .
\end{aligned}
$$

It takes the following form:

$$
\begin{aligned}
\mathcal{M}_{\mathrm{II}}^{\text {on }}= & \frac{\pi}{(2 \pi)^{3} \sqrt{4 \pi}} \sum_{m_{n} m_{p}} \frac{1}{2 P}\left(\frac{1}{2} m_{n} \frac{1}{2} m_{p} \mid 1 M\right) \\
& \times \int_{0}^{2 \pi} d \phi \int_{\left|p_{\min }(p \pi)\right|}^{p_{\max }(p \pi)} p u_{0}(p) d p \frac{m}{E_{p}}\left[T_{\gamma n} T_{\pi N}\right]_{q_{\pi}^{2}=m_{\pi}^{2}} \\
& +D \text { wave part. }
\end{aligned}
$$

The two-dimensional integral is done numerically. It depends on only on-shell elementary amplitudes. The weight $p u_{0}(p)$ selects nucleons almost at rest in the deuterium when the lower bound $p_{\min }(p \pi)$ of the integral vanishes. This is the origin of the meson-nucleon scattering peak in Fig. 1, which is therefore on solid ground.

The $\pi N$ scattering amplitude can be expressed as

$$
T_{\pi N}=\left(m_{1}\left|f\left(Q_{s}, t_{r}\right)+g\left(Q_{s}, t_{r}\right) \vec{\sigma} \cdot \vec{k}_{\perp}\right| m_{p}\right),
$$

where $t_{r}=\left(p_{\pi}-q_{\pi}\right)^{2}$ is the four-momentum transfer at the $\pi p$ rescattering vertex and $\vec{k}_{\perp}=\vec{p}_{\pi} \times \vec{q}_{\pi}$ is the direction perpendicular to the scattering plane. At high energies $\left(Q_{s}>\right.$ $2 \mathrm{GeV}$ ) the central part dominates at forward angles and is almost entirely absorptive. It can be parametrized as follows:

$$
f\left(Q_{s}, t_{r}\right)=-\frac{Q_{s} p_{\mathrm{c} . \mathrm{m} .}}{m}(\epsilon+i) \sigma_{\pi^{-} p} \exp \left(\frac{\beta_{\pi}}{2} t_{r}\right) .
$$

Above $Q_{s} \sim 2 \mathrm{GeV}$, the total cross section stays constant at the value $\sigma_{\pi^{-}} p=30 \mathrm{mb}$ [24], and the fit of the differential cross section at forward angles leads to a slope parameter $\beta_{\pi}=$ $6 \mathrm{GeV}^{-2}$ [23]. At high energy the ratio between the real and the imaginary parts of the amplitude is small [24], and I set it to zero in this study.

With such an absorptive amplitude it is easy to see from Eqs. (2) and (10) that the singular part of the rescattering amplitude interferes destructively with the quasi-free amplitude.

The principal part of the rescattering integral takes the following form:

$$
\begin{aligned}
\mathcal{M}_{\mathrm{II}}^{\mathrm{off}}= & \frac{i}{(2 \pi)^{3} \sqrt{4 \pi}} \sum_{m_{n} m_{p}}\left(\frac{1}{2} m_{n} \frac{1}{2} m_{p} \mid 1 M\right) \\
& \times \oint \frac{d^{3} \vec{p} u_{0}(p) T_{\gamma n} T_{\pi N}}{q_{\pi}^{2}-m_{\pi}^{2}} \frac{m}{E_{p}}+D \text { wave part. }
\end{aligned}
$$

It turns out that it vanishes $[1,30]$ when $p_{\min }(p \pi)=0$ at the top of the $\pi N$ rescattering peak and contributes little to its tails only, as in Fig. 1 for instance.

Since the Regge amplitude $T_{\gamma n}$ varies rapidly as $s^{\alpha\left(t_{f}\right)}$, with the total energy $s=\left(E_{2}+E_{q}\right)^{2}-\left(\vec{p}_{2}+\vec{q}_{\pi}\right)^{2}$ and momentum transfer $t_{f}=\left(k-q_{\pi}\right)^{2}$, it cannot be factorized out of the integral, which should be evaluated numerically. This is not a problem for its singular part: It is a twofold integral that involves well-defined on-shell quantities. Its principal part is a threefold integral that requires a good knowledge of the off-shell extrapolation of the elementary amplitude. Since its contribution is small near the singularity, I do not take it into account in this study in order to save time in the Monte Carlo simulation in the full phase space (Sec. II C). It will be taken be into account later, in the final analysis of experimental data. 


\section{Nucleon-nucleon rescattering}

The matrix element of the proton-proton rescattering amplitude [graph (III) in Fig. 2] takes the following form:

$$
\begin{aligned}
\mathcal{M}_{\mathrm{III}}\left(\vec{k}, \epsilon, M, \vec{p}_{\pi}, \vec{p}_{1}, m_{1}, \vec{p}_{2}, m_{2}\right) \\
=i \sum_{m_{n} m_{p} m_{p}^{\prime}}\left(\frac{1}{2} m_{n} \frac{1}{2} m_{p} \mid 1 M\right) \int \frac{d^{3} \vec{p}}{(2 \pi)^{3}} \frac{u_{0}(p)}{\sqrt{4 \pi}} \frac{1}{p^{\circ \prime}-E_{p}^{\prime}+i \epsilon} \\
\quad \times \frac{m}{E_{p}} T_{\gamma n}\left(\vec{p}^{\prime}, m_{p}^{\prime},-\vec{p}, m_{n}\right) \\
\quad \times T_{p p}\left(\vec{p}_{2}, m_{2}, \vec{p}_{1}, m_{1}, \vec{p}^{\prime}, m_{p}^{\prime}, \vec{p}, m_{p}\right)+D \text { wave part. }
\end{aligned}
$$

The integral runs on the three-momentum of the spectator proton in the loop, which has been put on-shell, $p^{0}=E_{p}=$ $\sqrt{\vec{p}^{2}+m^{2}}$, by the integration over its energy $p^{0}$. It can be split into two parts:

$$
\mathcal{M}_{\text {III }}=\mathcal{M}_{\mathrm{III}}^{\text {on }}+\mathcal{M}_{\mathrm{III}}^{\text {off }}
$$

The singular part of the rescattering integral runs between the minimum and the maximum values of the momentum of the spectator proton in the loop for which the struck proton can propagate on-shell:

$$
\begin{aligned}
& p_{\min }(p p)=\frac{P}{W} E_{\text {c.m. }}-\frac{E}{W} p_{\text {c.m. }}, \\
& p_{\max }(p p)=\frac{P}{W} E_{\text {c.m. }}+\frac{E}{W} p_{\text {c.m. } .}
\end{aligned}
$$

where $E=E_{2}+E_{1}, \vec{P}=\vec{p}_{2}+\vec{p}_{1}$, and $W=\sqrt{E^{2}-\vec{P}^{2}}$ are, respectively, the energy, the momentum, and the mass of the scattering $p p$ pair. The momentum and the energy of the spectator proton in the c.m. frame of the $p p$ pair are

$$
\begin{aligned}
& p_{\text {c.m. }}=\frac{\sqrt{W^{2}-4 m^{2}}}{2}, \\
& E_{\text {c.m. }}=\sqrt{p_{\text {c.m. }}^{2}+m^{2}}=\frac{W}{2} .
\end{aligned}
$$

As in the previous subsection, the singular part of the integral picks the low momentum components of the deuteron wave function, relies on on-shell elementary matrix elements, and is maximum when $p_{\min }(p p)=0$. The principal part vanishes under the rescattering peak and contributes little to its tails: The situation is the same as in the $n p$ rescattering sector of the ${ }^{2} \mathrm{H}\left(e, e^{\prime} p\right) n$ reaction (see, e.g., Fig. 2 of [17]).

The dependency on $t=\left(k-p_{\pi}\right)^{2}$ of the elementary photoproduction amplitude $T_{\gamma n}$ is fixed by the external kinematics [see graph (III) in Fig. 2] and not by the internal kinematics in the loop integral. Therefore it can be safely factorized out of the integrals and evaluated assuming that the target nucleon is at rest in the deuteron, in which case $s=2 m v+m^{2}$. The integrals can be performed analytically, following the method outlined in [30]. I have checked [17] that this approximation is very close (within $10 \%$ ) to the full evaluation of the integrals in the rescattering peak region. This saves computing time, and both the singular and principal parts have been retained: The proton rescattering peak is therefore slightly wider than the pion rescattering peak in Fig. 1.
The proton-proton scattering amplitude is taken as

$$
\begin{aligned}
T_{p p}= & \left(m_{2} m_{1} \mid \alpha+i \gamma\left(\vec{\sigma}_{1}+\vec{\sigma}_{2}\right) \cdot \vec{k}_{\perp}\right. \\
& \left.+ \text { spin }- \text { spin terms } \mid m_{p}^{\prime} m_{p}\right),
\end{aligned}
$$

where $\vec{k}_{\perp}$ is the unit vector perpendicular to the scattering plane.

Above $500 \mathrm{MeV}$, the central part $\alpha$ dominates. It is almost entirely absorptive and takes the following simple form;

$$
\alpha=-\frac{W p_{\text {c.m. }}}{2 m^{2}}(\epsilon+i) \sigma_{N N} \exp \left(\frac{\beta_{N}}{2} t_{r}\right),
$$

where $t_{r}=\left(p^{\prime}-p_{1}\right)^{2}$ is the four-momentum transfer at the $p p$ scattering vertex. In the forward direction its imaginary part is related to the total cross section $\sigma_{N N}$, while the slope parameter $\beta_{N}$ is related to the angular distribution of $N N$ scattering at forward angles. I use the same values as in [17]. Note that the difference in the norm of Eqs. (21) and (12) comes from the choice of the norm of the spinors, $\bar{u} u=1$.

\section{B. Coplanar kinematics}

Figure 1 exhibits the salient predictions of the model. It corresponds to coplanar kinematics that can be achieved by detection of the pion and one of the protons with two well-shielded magnetic spectrometers in Hall A or Hall C at JLab for instance. It shows the ratio of the full cross section to the quasi-free cross section as a function of the polar angle of the slow nucleon $\theta_{R}=\theta_{1}$ when its momentum $P_{R}=\left|\vec{p}_{1}\right|$ is kept constant at $200 \mathrm{MeV} / c$ (lower curve) or $500 \mathrm{MeV} / c$ (upper curve). The mass of the pair made of the pion and the fast (second) nucleon is kept constant at the value $W=$ $\sqrt{\left(p_{2}+p_{\pi}\right)^{2}}=2.896 \mathrm{GeV}$ that corresponds to the absorption of a $4-\mathrm{GeV}$ photon by a nucleon at rest. The four-momentum transfer is also kept constant at the value $t=\left(k-p_{\pi}\right)^{2}=$ $-3 \mathrm{GeV}^{2}$, which corresponds to the emission of the pion around $90^{\circ}$ in the $\pi p_{2} \mathrm{c} . \mathrm{m}$. frame.

At high recoil momentum, rescattering mechanisms dominate the quasi-free contribution. The top of the peaks corresponds to kinematics in which an on-shell pion or nucleon can be produced on a nucleon at rest $\left(p_{\min }=0\right)$ in the rescattering amplitude. The width of the peaks reflects the Fermi motion of the target nucleon. The physical picture is as follows. The pion (respectively, proton) is photoproduced on a neutron at rest in deuterium, propagates on-shell, and rescatters on the spectator proton, also at rest in deuterium. Two-body kinematics requires that the angle between the scattered pion (proton) and the recoiling proton be constant (strictly $90^{\circ}$ for $p p$ elastic scattering). Since the recoiling nucleon momentum is fixed, the angles that it makes with the total momenta $\vec{p}_{1}+\vec{p}_{\pi}$ or $\vec{p}_{1}+\vec{p}_{2}$ are also fixed: Typically $70^{\circ}$. Therefore, the $\pi p$ or the $p p$ rescattering peaks form a cone centered along the direction of the total momentum of the corresponding scattering pair. In coplanar kinematics, two peaks appear for each rescattering, depending on whether or not the pion and the recoiling proton are emitted on the same side of the photon.

The difference in the height of each of these two peaks reflects the rapid variation with the photon energy of the elementary pion photoproduction Regge cross sections: $s^{2 \alpha(t)-2}$. 


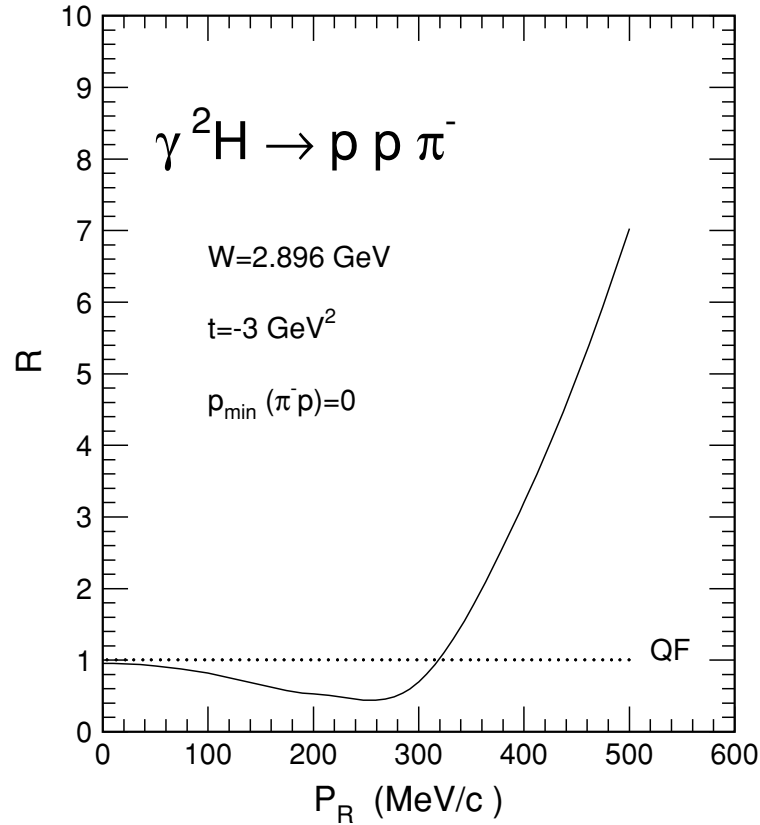

FIG. 4. Ratio of the total to the quasi-free cross section of the ${ }^{2} \mathrm{H}\left(\gamma, \pi^{-} p\right) p$ reaction against the momentum of the recoiling proton at the top of the $\pi p$ rescattering peak. The solid curve includes $\pi p$ scattering and $p p$ scattering (small effect). The dotted line corresponds to the quasi-free process.

Although the mass of the pair made of the pion and the fast proton has been kept constant in Fig. 1, the incoming photon must also provide the energy of the slow recoiling nucleon: This depends on its direction of motion. For instance, at the top of the $\pi p$ peak that is located on the left-hand side in Fig. 1 the photon energy is $E_{\gamma}=3.432 \mathrm{GeV}$, while it is $E_{\gamma}=$ $5.436 \mathrm{GeV}$ at the top of the peak at the right. Since, in the rescattering amplitude, the photoproduction occurs on a nucleon at rest the corresponding masses are, respectively, 2.705 and $3.328 \mathrm{GeV}$. The situation is the same at the top of the two $p p$ rescattering peaks.

At low recoil momentum, this effect is less dramatic since the energy difference is less important (it vanishes at $P_{r}=0$ !). Here the rescattering amplitudes interfere destructively with the quasi-free amplitude, consistently with unitary. Since the elementary $\pi p$ and $p p$ scattering amplitudes are dominantly absorptive in the energy range covered by this study, a part of the strength is shifted from the quasi-free channel to inelastic channels. Above $p_{r}=300 \mathrm{MeV} / c$, rescattering contributions take over and dominate the cross section. Figure 4 shows this evolution of the cross section at the top of the $\pi p$ rescattering peak $\left(\theta_{R}=-50^{\circ}\right.$ in Fig. 1$)$ with the recoil momentum $P_{R}$.

\section{CLAS kinematics}

The CLAS [16] set up at JLab allows us to record events in the full available phase space and is well suited to make a survey of the cross section of the ${ }^{2} \mathrm{H}\left(\gamma, p \pi^{-}\right) p$ reaction and to exploit its features, which we just discussed.

Three superconducting coils generate a toroidal field perpendicular to the photon-beam axis and define six sectors where particles are detected by wire chambers and scintillators. The geometrical fiducial acceptance represents more than $2 \pi \mathrm{sr}$. It covers a range of polar angles between $11^{\circ}$ and $140^{\circ}$, but the coils define six azimuthal regions where the detector is blind.

I implemented the code that computes the cross section of the ${ }^{2} \mathrm{H}\left(\gamma, p \pi^{-}\right) p$ reaction in a Monte Carlo code that generates events in the full fiducial acceptance of the CLAS. I sampled, with a flat distribution, the three-momentum $\vec{p}_{1}$ of the slow proton and the two angles $\cos \theta_{2}$ and $\phi_{2}$ of the fast proton. If each proton fell in the fiducial acceptance, which I took from Ref. [32], I recorded the kinematics of the event in a database (namely an $N$-tuple in the CERN package PAW [33]), and I weighted it with the corresponding differential cross section

$$
\frac{d \sigma}{d p_{1} d \Omega_{p_{1}} d \Omega_{p_{2}}}=J \times \frac{d \sigma}{d \vec{p}_{1}\left[d \Omega_{\pi}\right]_{\mathrm{c} . \mathrm{m} .2}},
$$

where $J$ is the relevant Jacobian:

$$
J=\frac{Q_{f}\left|\vec{p}_{2}\right|^{3}\left|\vec{p}_{1}\right|^{2}}{\left.\mu_{\mathrm{c} . \mathrm{m} .}\left|E_{\pi}\right| \vec{p}_{2}\right|^{2}-E_{2} \vec{p}_{\pi} \cdot \vec{p}_{2} \mid}
$$

(see Sec. II A for the definition of momenta and energies).

The events in the database are then binned as the experimental data, with the same cuts. This is the most straightforward way to compare a theory with experiments or to simulate experiments that are carried out over a wide and complicated phase space.

Figure 5 shows various observables that emphasize the pion-nucleon rescattering sector. The real photon-beam end point has been set to $E_{\gamma}=6 \mathrm{GeV}$, but the mass of the

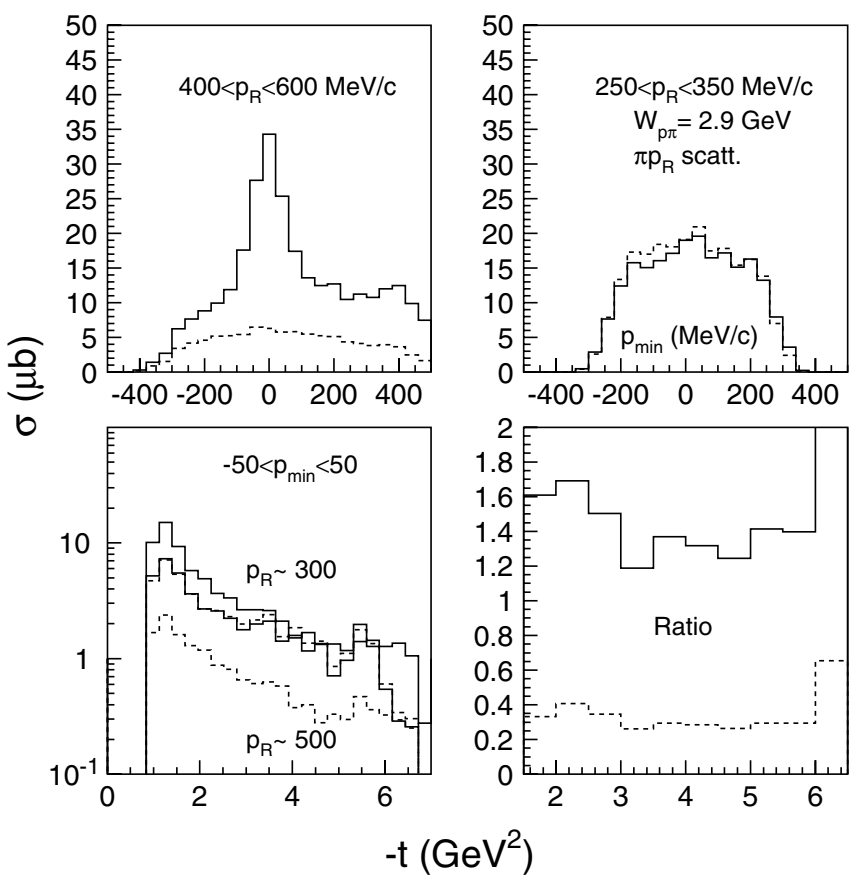

FIG. 5. CLAS kinematics for $\pi p$ rescattering in the ${ }^{2} \mathrm{H}\left(\gamma, p p_{R}\right) \pi^{-}$reaction. The beam end point is $6 \mathrm{GeV}$. The full histograms correspond to the full calculation, while the dashed histograms correspond to the quasi-free process only. See text for the description of the cuts that have been used in each window. 
fast proton-pion pair has been restricted to the range $2.85<$ $W_{p_{2} \pi}<2.95 \mathrm{GeV}$ to correspond to Fig. 1. Only events corresponding to large momentum transfer $-t>1 \mathrm{GeV}^{2}$ have been retained. The panel shows cross sections integrated over the various bins within the CLAS fiducial acceptance. The top parts show the distribution of the minimum momentum $p_{\min }\left(p_{1} \pi\right)$, Eq. (6), of the spectator proton in the pion-nucleon scattering loop for which the pion can propagate on-shell. On the left-hand side, the cut $400<P_{R}<600 \mathrm{MeV} / \mathrm{c}$ was applied on the momentum $p_{1}$ of the slow nucleon: The pion-nucleon rescattering peak clearly appears at $p_{\min }=0$. On the right-hand side, the cut $250<p_{R}<350 \mathrm{MeV} / c$ was applied: Rescattering effects are small here, consistent with Fig. 4, and the shape of the distribution reflects the kinematics and the detector acceptance. This is a good reference point that emphasizes the quasi-free process.

A further cut $-50<p_{\min }\left(p_{1} \pi\right)<50 \mathrm{MeV} / c$ was applied in the bottom parts of Fig. 5: It emphasizes pion nucleon rescattering. The $t$ distribution is plotted on the left-hand side for either low recoil momentum $(\sim 300 \mathrm{MeV} / c)$ or high recoil momentum $(\sim 500 \mathrm{MeV} / c)$ bands. The ratio of the second $\left(p_{R}=500 \mathrm{MeV} / c\right)$ to the first $\left(p_{R}=300 \mathrm{MeV} / c\right) t$ distribution is plotted on the right-hand side. In the plane wave approximation, it is nothing but the ratio of these high momentum to low momentum components of the nucleon momentum distribution in deuterium. The full ratio is really a measure of the evolution of the top of the pion-nucleon rescattering peak with the four-momentum transfer $t$, which fixes the hard scale. It is almost flat (the oscillations are due to the statistical accuracy of the Monte Carlo sampling) and provides us with a good starting point to look for deviations, especially at high $-t$, which could reveal the onset of color transparency for instance. The last bins $\left(-t>6 \mathrm{GeV}^{2}\right)$ in the $t$ distribution should be disregarded since they correspond to the kinematical limits where the detector acceptance differs strongly at low and high recoil momentum.

Figure 6 shows the same observables in the proton-proton rescattering sector. Now the minimum momentum $p_{\min }\left(p_{2} p_{1}\right)$ is the lowest value, Eq. (16), of the momentum of the spectator proton for which the other proton can propagate on-shell in the nucleon-nucleon scattering loop. Again, the bins at the highest values of $-t$ should be disregarded since they lie at the kinematics limits. Also, the statistical accuracy can be improved if the Monte Carlo code is run with more events (but also longer!).

In Fig. 5, $p p$ rescattering gives also a contribution below the $p \pi$ rescattering peak. Also, $p \pi$ rescattering gives a contribution under the $p p$ rescattering peak in Fig. 6. One can remove these contaminations by cutting the overlapping region in the joint distribution of the rescattering singularities, which is shown in Fig. 7.

In the Monte Carlo simulation, the choice was made to detect the two protons. The advantage is that the efficiency for detecting a proton in each sector of the CLAS is very good (better than $90 \%$, and only a small correction has to be applied to the histograms before comparing them with experiment): This is particularly interesting when one selects events corresponding to a large recoil momentum, of which the probability is small. However, this prevents recording
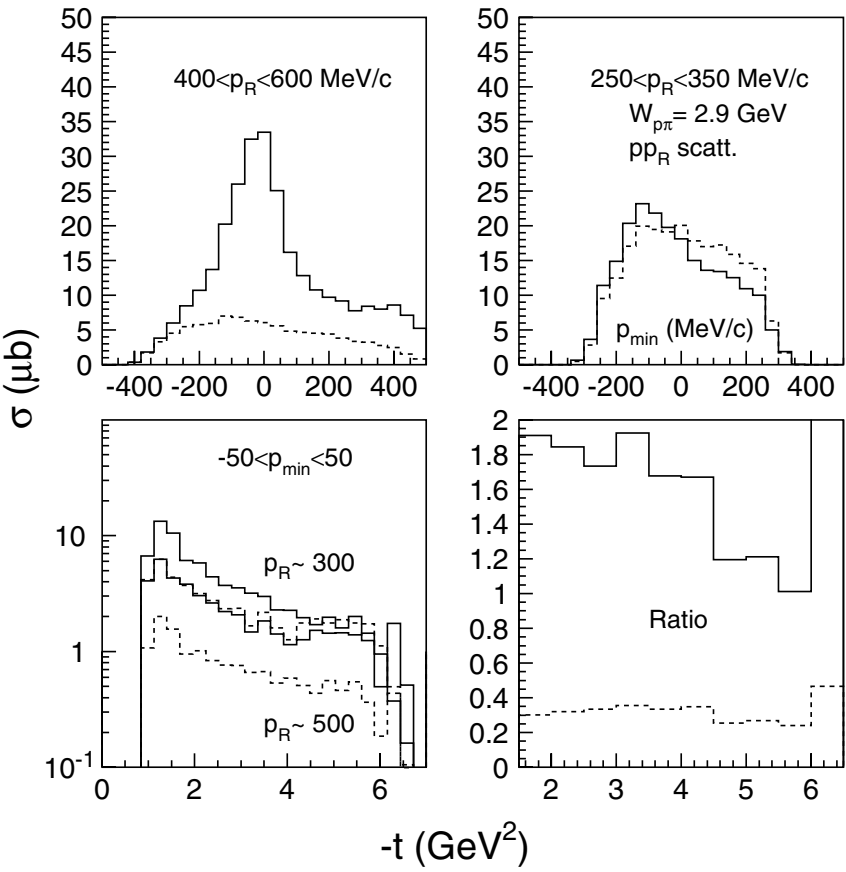

FIG. 6. CLAS kinematics for $p p$ rescattering in the ${ }^{2} \mathrm{H}\left(\gamma, p p_{R}\right) \pi^{-}$reaction. The beam end point is $6 \mathrm{GeV}$. The full histograms correspond to the full calculation, while the dashed histograms correspond to the quasi-free process only. See text for the description of the cuts that have been used in each window.

events with small recoil momentum, since CLAS cannot detect protons with a momentum lower than $\sim 250 \mathrm{MeV} / c$. For recording such events one must detect the $\pi^{-}$, which is bent inward, in the beam direction, by the magnetic field and also by decay in flight. Its detection efficiency is much smaller

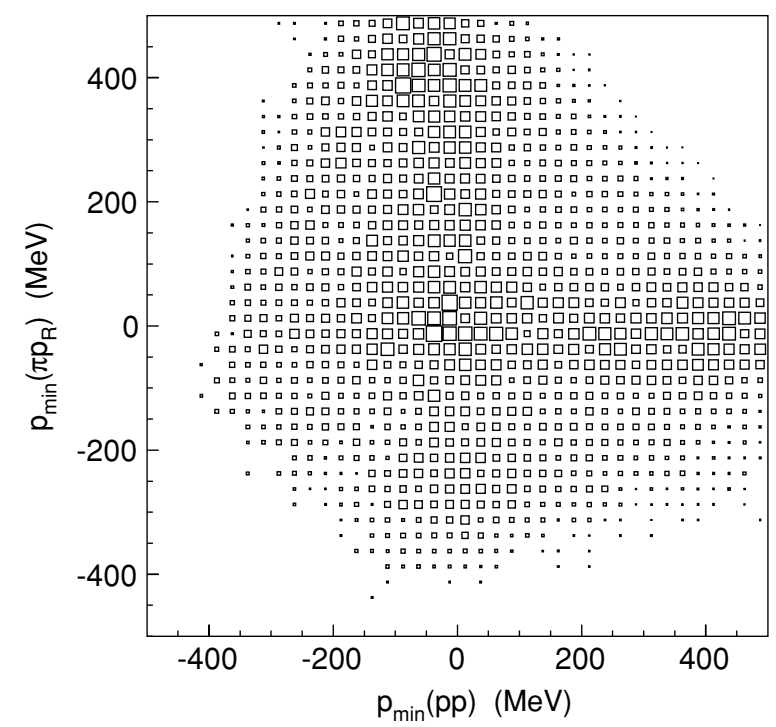

FIG. 7. The joint distribution of singularities in $p p$ and $p \pi$ rescattering in the ${ }^{2} \mathrm{H}\left(\gamma, p p_{R}\right) \pi^{-}$reaction. The beam end point is $6 \mathrm{GeV}$. The range of mass of the fast $p \pi$ pair is $2.85<W_{p_{2} \pi}<2.95 \mathrm{GeV}$. The range of the four-momentum transfer is $-t>1 \mathrm{GeV}^{2}$. The range of the momentum of the slow proton is $400<P_{R}<600 \mathrm{MeV} / c$. 
than that of a proton, but on the other hand the cross section is higher at low recoil momentum.

\section{Determination of the $\pi^{-}$elementary production amplitude}

The preceding discussion relies on good knowledge of the cross section of the elementary reaction $n\left(\gamma, \pi^{-}\right) p$. It can be determined in the same data set [27], since no free-neutron target exists. To that end, one has to detect the $\pi^{-}$and the fast proton $p_{2}$ and restrict the analysis to small values of the spectator slow proton, say, $p_{1}<100 \mathrm{MeV} / c$.

Figure 8 shows that rescattering corrections are less than $10 \%$ up to recoil momentum around $50 \mathrm{MeV} / c$. Above, the effects are larger. The neutron cross section is deduced from the deuterium cross section as follows

$$
\left(1+\beta_{1} \cos \theta_{1}\right) \frac{d \sigma}{d \Omega_{\pi}}=\frac{d \sigma}{d \vec{p}_{1} d \Omega_{\pi}} \times \frac{1}{\rho\left(\left|\vec{p}_{1}\right|\right)},
$$

where $\rho\left(\left|\vec{p}_{1}\right|\right)$ is the neutron momentum distribution in the deuteron. I kept the flux factor in order to quantify the corresponding slope of the quasi-free cross section.

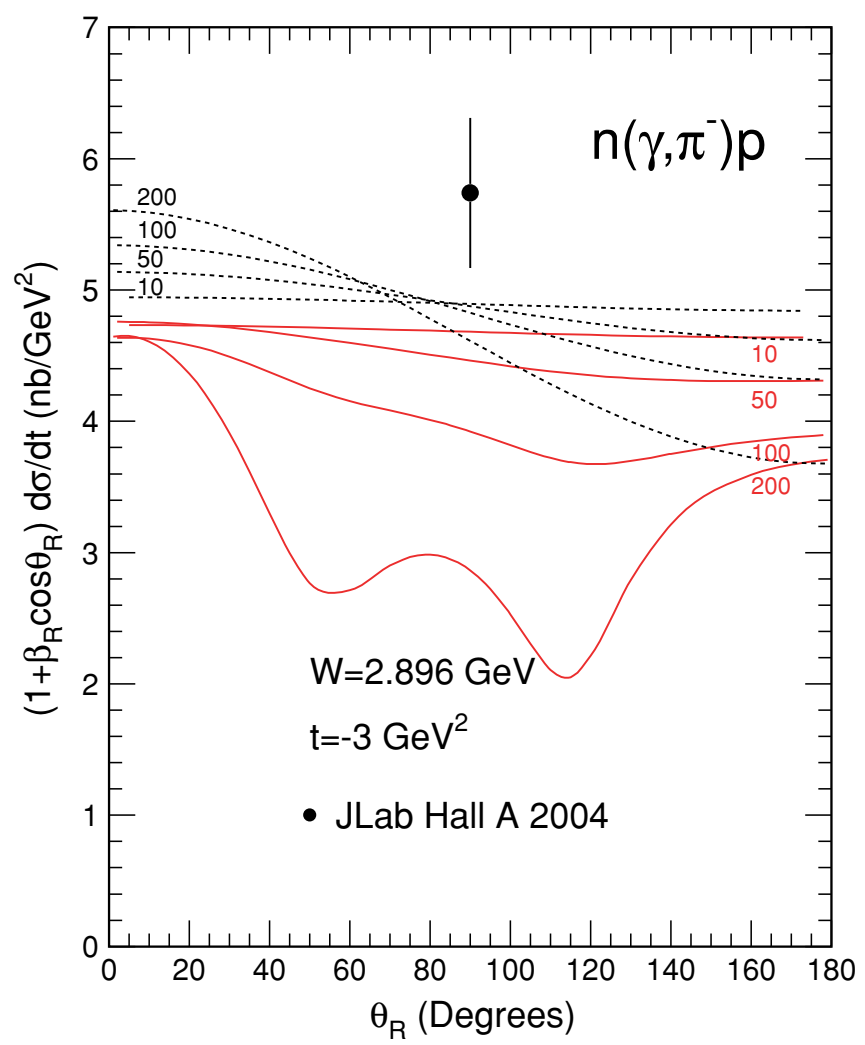

FIG. 8. (Color online) The c.m. cross section of the $n\left(\gamma, \pi^{-}\right) p$ reaction as extracted from the analysis of the ${ }^{2} \mathrm{H}\left(\gamma, \pi^{-} p\right) p$ reaction when the angle of the recoiling proton varies but its momentum is kept constant at 10,50,100, and $200 \mathrm{MeV} / c$. The dotted curves correspond to the quasi-free process. The kinematics is coplanar, and only the part of the angular distribution that corresponds to the emission of the pion and the recoiling proton on different sides of the photon is shown. Each curve is labeled with the value of the corresponding recoil momentum; on the left-hand side for the quasi-free result, on the right-hand side for the full calculation.
The kinematics, $W=2.896 \mathrm{MeV}$ and $t=-3 \mathrm{GeV}^{2}$, corresponds to one of the few data points [31] recently measured in Hall A at JLab. It corresponds to the kinematics in Fig. 1, as well as to the average kinematics covered by this study, and indicates that the elementary amplitude is not far off the mark. This experimental datum (and a few others) has been obtained from the analysis of the ${ }^{2} \mathrm{H}\left(\gamma, \pi^{-}\right)$reaction induced by a Bremsstrahlung photon beam, which averages the elementary cross section over the Fermi motion of the neutron in the deuterium target. Since the major contribution comes from neutron momentum in the range of $\sim 80 \mathrm{MeV} / c$, interaction effects cannot be neglected. The analysis of the high-statistics Hall B experiment [27], along the line of Fig. 8, would be very welcome for enlarging the data set and improving its accuracy.

To correspond to Fig. 1 , the cross sections at $p_{R}=p_{1}=$ $200 \mathrm{MeV} / \mathrm{c}$ are also plotted in Fig. 8. The ratio of the full calculation to the quasi-free one is plotted in Fig. 1. When multiplied by the momentum distribution $\rho\left(\left|\vec{p}_{1}\right|\right)$, these curves become the fully differential cross sections $d \sigma / d \vec{p}_{1} d \Omega_{\pi}$. One notes that the quasi-free cross sections follow almost exactly the variation of the flux factor $1+\beta_{1} \cos \theta_{1}$, but decrease a little when the recoil momentum $p_{1}$ increases by $2 \%$ at $100 \mathrm{MeV} / c$, by $5 \%$ at $200 \mathrm{MeV} / c$, and by no more than $10 \%$ above. This is a consequence of the choice of the off-shell extrapolation of the elementary pion-photoproduction amplitude (see appendix) and a measure of the uncertainty on the determination of the quasi-free amplitude at high recoil momenta. However, rescattering amplitudes dominate here (by about a factor 5 and more, at the top of the rescattering peak), and they are driven by on-shell elementary matrix elements and the low momentum components of the nuclear wave function. Therefore the uncertainties in the off-shell extrapolation of the elementary amplitudes do not affect the cross section in the domains of the rescattering peaks.

\section{THE STRANGE SECTOR}

The extension to the ${ }^{2} \mathrm{H}\left(\gamma, K^{+} \Lambda\right) n$ reaction is straightforward. The amplitude of the elementary reaction $p\left(\gamma, K^{+}\right) \Lambda$ is driven by the exchange of the Regge trajectories, of the $K$ and the $K^{*}$ [22]. Besides trivial changes in the mass of the particles, the Regge trajectories, and the coupling constants, the reaction amplitudes exhibit the same form as in the pion-production sector (Section II).

The kaon is produced on the proton. Since the neutron and the $\Lambda$ in the final state are distinct particles, there is no need to antisymmetrize the reaction amplitude.

All the coupling constants and Regge propagators are given in Ref. [22]. I use the slope parameters $\beta_{K}=3 \mathrm{GeV}^{-2}$ [23] and $\beta_{\Lambda}=\beta_{p n}$ [17] and the following cross sections [24]: $\sigma_{K^{+} n}=18 \mathrm{mb}$ and $\sigma_{\Lambda n}=35 \mathrm{mb}$ in the $K^{+} n$ and $\Lambda n$ scattering amplitudes, respectively. However, these parameters are less known than in the $\pi N$ and $N N$ scattering sectors, and their choice should be refined by the analysis of the ${ }^{2} \mathrm{H}\left(\gamma, K^{+} \Lambda\right) n$ reaction at a low four-momentum transfer $t$.

Figure 9 shows the ratio of the full cross section to the quasi-free cross section as a function of the polar angle of the slow neutron, $\theta_{R}=\theta_{n}$, when its momentum $P_{R}=|\vec{n}|$ is kept 


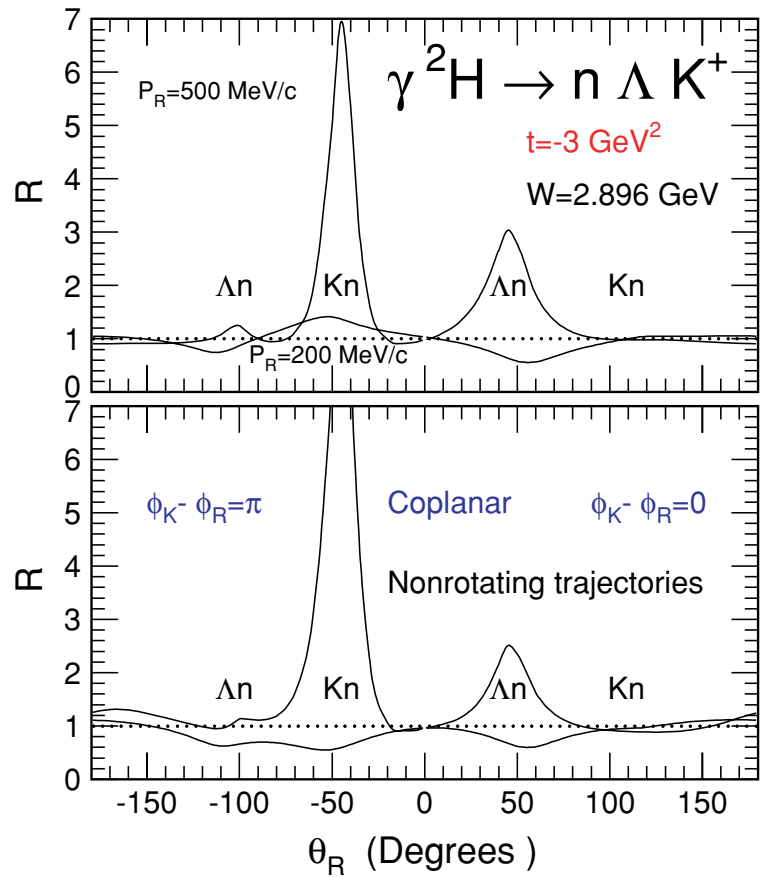

FIG. 9. (Color online) Ratio of the total to the quasi-free cross section of the ${ }^{2} \mathrm{H}\left(\gamma, K^{+} \Lambda\right) n$ reaction against the angle of the recoiling neutron whose momentum is kept constant at $500 \mathrm{MeV} / \mathrm{c}$ (top curves) and $200 \mathrm{MeV} / c$ (bottom curves). The peaks labeled $K^{+} n$ and $\Lambda n$ correspond respectively to $K^{+} n$ rescattering and $\Lambda n$ on-shell rescattering. The dotted lines correspond to the quasi-free process. The kinematics is coplanar, and positive angles correspond to the emission of the kaon and the recoiling neutron on the same side of the photon. The top panel corresponds to a rotating phase, while the bottom panel corresponds to no phase in Regge trajectories.

constant at $200 \mathrm{MeV} / c$ (lower curve) or $500 \mathrm{MeV} / c$ (upper curve). The mass of the pair made of the $K^{+}$and the $\Lambda$ is kept constant at the value $W=\sqrt{\left(p_{\Lambda}+p_{K^{+}}\right)^{2}}=2.896 \mathrm{GeV}$ that corresponds to the absorption of a $4-\mathrm{GeV}$ photon by a nucleon at rest. The four-momentum transfer is also kept constant at the value $t=\left(k-p_{K^{+}}\right)^{2}=-3 \mathrm{GeV}^{2}$, which corresponds to the emission of the kaon around $90^{\circ}$ in the $K^{+} \Lambda$ c.m. frame.

At high recoil momentum, the pattern is the same as in the pion-production sector (Fig. 1). The heights of the rescattering peaks are different simply because the hadronic cross sections and their slopes are different. At low recoil momentum, however, the pattern is different in the $K n$ scattering sector. The reason is that, contrary to the $n\left(\gamma, \pi^{-}\right) p$ reaction Regge amplitudes, the $p\left(\gamma, K^{+}\right) \Lambda$ reaction Regge amplitudes exhibit a phase $\exp [-\imath \pi \alpha(t)]$ (see the discussion in Sec. 2.3.2 of Ref. [22]). In the $K n$ rescattering integral, which selects nucleons almost at rest in deuterium, the average four-momentum transfer $t$ is different than in the quasi-free production amplitude, where the target nucleon moves with a momentum fixed by the kinematics. This difference in $t$ changes the phase and compensates for the destructive interference between the quasi-free amplitude and the absorptive rescattering amplitude, around $200 \mathrm{MeV} / c$.

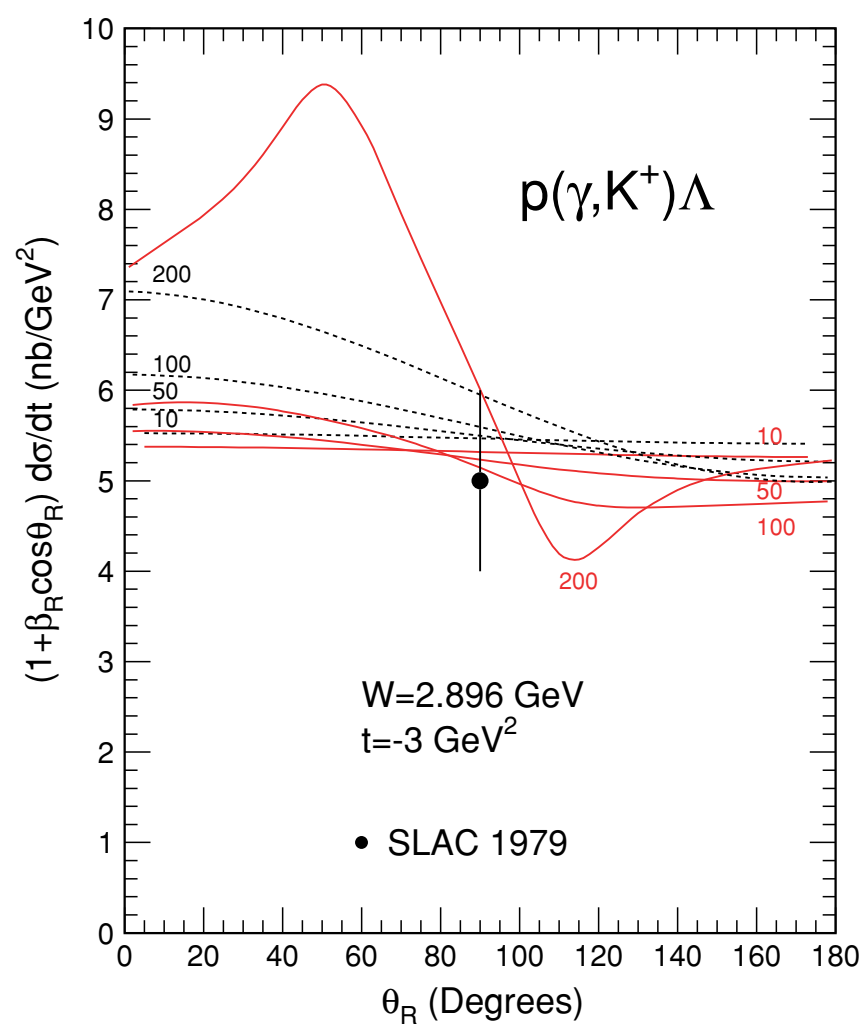

FIG. 10. (Color online) The c.m. cross section of the $p\left(\gamma, K^{+}\right) \Lambda$ reaction as extracted from the analysis of the ${ }^{2} \mathrm{H}\left(\gamma, K^{+} \Lambda\right) n$ reaction when the angle of the recoiling neutron varies but its momentum is kept constant at 10,50,100, and $200 \mathrm{MeV} / c$. The dotted curves correspond to the quasi-free process. The kinematics is coplanar, and only the part of the angular distribution that corresponds to the emission of the kaon and the recoiling neutron on different sides of the photon, is shown. Each curve is labeled with the value of the corresponding recoil momentum; on the left-hand side for the quasi-free result, on the right-hand side for the full calculation.

When the Regge phases are turned off, in the bottom part of Fig. 9 , the pattern becomes the same as in the $\pi^{-}$production channel in Fig. 1.

This effect does not occur in the $\Lambda n$ rescattering sector, since $t$ is defined by the external kinematics and is the same in the rescattering amplitude and the quasi-free amplitude (see Fig. 2).

Figure 10 shows the value of the elementary kaonphotoproduction cross section as extracted from a deuteron target with Eq. (24) for different values of the recoiling neutron. As in the pion-production sector, the quasi-free cross section follows the variation of the flux factor $1+\beta_{1} \cos \theta_{1}$ and departs from the free cross section $\left(p_{R}=10 \mathrm{MeV} / c\right)$ when the recoil momentum increases. The datum in Fig. 10 is one of the few experimental cross sections available at high momentum transfer [34].

The reaction amplitudes rely on the elementary photoproduction of a kaon on a proton target. The Regge model leads to a fair agreement with the existing set of data around $E_{\gamma}=$ $4 \mathrm{GeV}$ (see [22]). This data set can be greatly enlarged by the analysis of the high statistical accuracy experiment [35] 
recently completed in CLAS, with a beam of a real photon of $4 \mathrm{GeV}$ on a proton target.

To achieve the full exclusivity of kaon production off a deuterium target, one needs to detect both the $K^{+}$and the $\Lambda$ which can be identified by its decay into a $\pi^{-} p$ pair. The CLAS is ideally suited to record such a three-charged-particle configuration. When the $\Lambda$ decay distribution is implemented in the Monte Carlo code, the simulation leads to histograms similar to those which have been obtained in the pion sector. Since the physical content is the same, they are not shown but will be compared with experiment with the same cuts when the analysis is completed.

Other channels can also be studied. Of particular interest is the ${ }^{2} \mathrm{H}\left(\gamma, K^{0} \Lambda\right) p$ reaction [36] in which all the (decay) particles in the final state are charged. As in the $\pi^{-}$sector the cross section of the elementary reaction $n\left(\gamma, K^{0}\right) \Lambda$ must be determined from the same data set, demanding a low momentum $(p<50 \mathrm{MeV} / c)$ spectator proton. Also the ${ }^{2} \mathrm{H}\left(\gamma, K^{+} \Lambda^{*}\right) n$ channel should be considered, since a Regge model based on the exchange of the $K$ and $K^{*}$ mesons [37] leads also to a good agreement of the differential cross section of the elementary reaction $p\left(\gamma, k^{+}\right) \Lambda^{*}$ at $E_{\gamma}=3.5 \mathrm{GeV}$. Any signal in the $K^{+} n$ scattering sector should be the same as in the ${ }^{2} \mathrm{H}\left(\gamma, K^{+} \Lambda\right) n$ reaction.

\section{VECTOR-MESON PRODUCTION}

Exclusive vector-meson production on few body systems is certainly very promising. It allows us to prepare a pair of quarks, with an adjustable transverse size, and to study its interaction with a nucleon in well-defined kinematics. Furthermore, the coherence time (during which the incoming photon oscillates into a $q \bar{q}$ pair) and the formation time (after which this pair recombines into the final meson) can be adjusted independently to the internucleonic distance.

A special emphasis should be put on $\phi$ and $J / \Psi$ mesons production: Not only are these narrow states more easy to identify, but their flavor content, different from that of the ground state of cold hadronic matter, makes them a promising probe.

Figure 11 shows the expected signal in the $\phi$ photoproduction channel when the photon beam end point is $E_{\gamma}=$ $6 \mathrm{GeV}$. The model is a straightforward extension of the previous amplitudes. Again, the mass of the $p \phi$ fast pair and the four-momentum transfer are set at $W_{p \phi}=2.896 \mathrm{MeV}$ and $t=\left(k-p_{\phi}\right)^{2}=-3 \mathrm{GeV}^{2}$, respectively. The elementary amplitude $[38,39]$ is based on the exchange of two nonperturbative gluons and uses a correlated nucleon wave function. It leads to a very good account of the $p(\gamma, \phi) p$ reaction [40] recently measured at JLab at $E_{\gamma}=4 \mathrm{GeV}$. The $\phi$ can be photoproduced on the proton as well as on the neutron: This has been taken into account in the model. The $p n$ scattering amplitude is defined according to Ref. [17], while the $\phi n$ total cross section and slope parameter are, respectively, $\sigma_{\phi n}=20 \mathrm{mb}$ and $\beta=$ $6 \mathrm{GeV}^{-2}$. Again those quantities are almost unknown and must be determined by the analysis of the ${ }^{2} \mathrm{H}(\gamma, \phi) p n$ reaction at low $-t$. The $\phi$ nucleon scattering cross section has been extracted

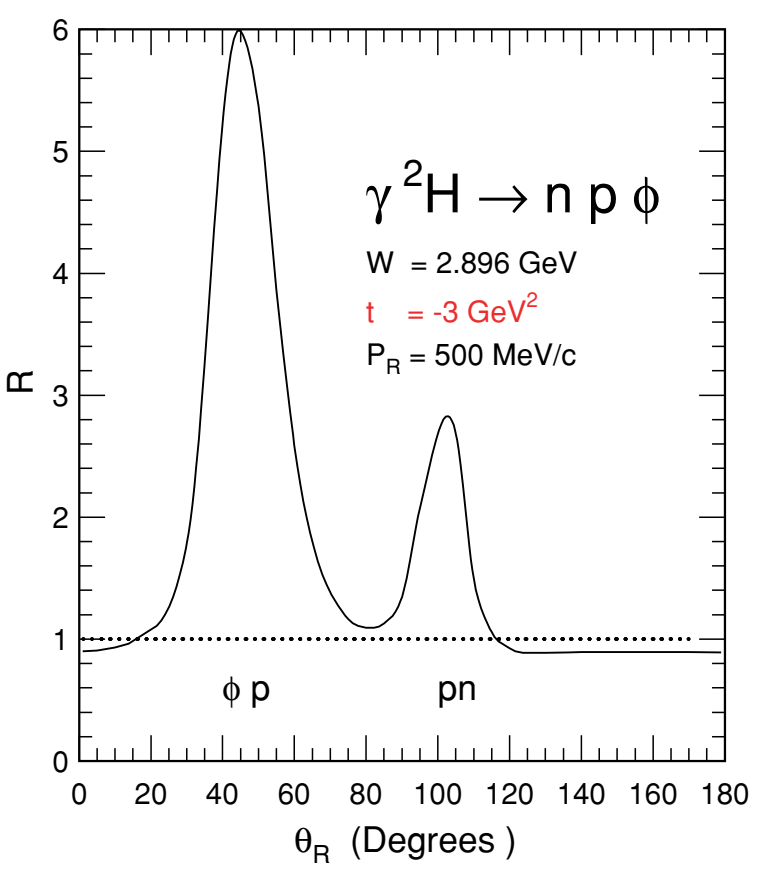

FIG. 11. (Color online) Ratio of the total to the quasi-free cross section of the ${ }^{2} \mathrm{H}(\gamma, \phi p) n$ reaction against the angle of the recoiling neutron whose momentum is kept constant at $500 \mathrm{MeV} / c$. The kinematics is coplanar and only the part that corresponds to the emission of the meson and the recoiling neutron on different sides of the photon is shown.

from one experiment (see Ref. [41]), while I have taken the universal slope for high-energy diffractive processes.

Such a study may end up giving us a better understanding of the formation of vector mesons in cold hadronic matter and will be a reference for the study of vector-meson production in heavy-ion collisions.

\section{COLOR TRANSPARENCY AND HADRON PROPAGATION}

The expected effect of color transparency would be a reduction of the rescattering peak in Figs. 5, 6, 9, and 11 when the four-momentum transfer $-t$ increases. The idea is that hard scattering mechanisms produce colorless dipoles with a small transverse size. Their scattering cross section is therefore expected to be reduced according to the square of the ratio of their transverse size to the transverse size of their asymptotic states.

In addition, the rescattering peaks are expected to be wider since the small configuration is not an eigenstate of the mass operator. It is rather a combination of particles that can be diffractly excited from the ejectile [42]. In the rescattering integrals [Eqs. (4) and (14)] the propagator of these excited states should be added to propagator of the meson or the baryon that rescatters. The corresponding singularities are closely related to the mass of each particle of the spectrum and lead to peaks at slightly different locations. The rescattering peaks will be spread according to the actual distribution of these states in the mass spectrum of the small configuration. 
In previous reviews or prospect talks $[11,25,26]$ I used a toy model based on a geometrical expansion of the mini configuration of the ejected hadron. Now it is superseded by the quantum diffusion model [42-45] that can be implemented in Eqs. (4) and (14). I defer this study until dedicated experiments are performed. Only experiments will tell us what is the relevant nature of the process that governs the formation and the evolution of such a small configuration.

This paper provides a baseline calculation under the assumption that normal particles rescatter. This is a unique situation that relies on the evolution of a peak rather than on the level of attenuation of a flat cross section.

The key parameter is the time scale of the interaction. If it is long, the degrees of freedom are hadrons, and well-defined unitary peaks appear according to the study presented in this paper. If it is short enough, the degrees of freedom are quarks or exotic objects and unitary peaks should be different or disappear.

Exclusive reactions at hight $t$ are certainly best suited to these studies: They define the small interaction volume for which quarks may be the relevant degrees of freedom. The virtuality $Q^{2}$ of the photon exchanged in electron scattering defines the volume of observation. Playing with these two independent hard scales is the key to the understanding of these rare processes [18]. It can be started at $6 \mathrm{GeV}$, but clearly higher energies ( $12 \mathrm{GeV}$ and even above) are needed.

\section{EXOTICS}

The rescattering peaks also offer us a tool to determine the cross section of the interaction with nucleon of unstable or exotic particles for which a beam is not available. Obvious examples are the photoproduction of strange baryons $(\Lambda, \Sigma \ldots)$, of vector mesons $(\phi, J / \Psi \ldots)$, etc.

They can also offer us a way to disentangle elusive exotics, such as pentaquarks, and the physical background. One way to chase pentaquarks has been to determine the variation of the mass of the $K^{+} n$ pair that is emitted in photoreactions induced on nuclear [46] or deuterium [47] targets. Selecting the $K^{+} n$ unitary rescattering peak, $\left|p_{\min }\left(n K^{+}\right)\right|<100 \mathrm{MeV} / c$ for instance, in the reaction ${ }^{2} \mathrm{H}\left(\gamma, K^{+} \Lambda\right) n$ or ${ }^{2} \mathrm{H}\left(\gamma, K^{+} \Lambda^{*}\right) n$ would be the best way to master the physical background in the mass distribution of the $K^{+} n$ pair.

The extension of the model presented in this paper is straightforward: Put simply, the high-energy description of the $K^{+} n$ scattering should be replaced with a low-energy description consistent with the existing data set. The contribution of a resonant state of a given width could be added in order to set a limit of the production cross section of a possible exotic. Also, the decay distribution of the $\Lambda$ or the $\Lambda^{*}$ should be taken into account in the Monte Carlo simulation of the experiment. I defer this study to a forthcoming paper [48].

\section{CONCLUSION}

At the top of each unitary rescattering peak, the reaction mechanism is well under control. It depends on on-shell elementary matrix elements and involves the low momentum components of the nuclear wave function. This is a good starting point to obtain the interaction with nucleons of exotic objects and short-lived particles.

This offers us a last chance to see and study color transparency in the present JLab energy range, more particularly in the strange quark sector. It also gives access to the determination of the cross sections of the scattering of vector and pseudo-scalar mesons on nucleons in a cold nuclear environment. Their knowledge is a key to the analysis of collisions between heavy ions at high energy.

For simplicity, all the numerical predictions in this study rely on a high-energy description of the elementary matrix element. The photoproduction amplitude is described by the exchange of the Regge trajectories of pseudoscalar and vector mesons. The hadron scattering amplitudes are almost entirely absorptive. This treatment is already valid at kinematics that is achievable at JLab at $6 \mathrm{GeV}$ : Mass of the meson nucleon system above $2 \mathrm{GeV}$, relative kinetic energy between baryons above $0.5 \mathrm{GeV}$. It will be even more valid in the kinematical range that will be accessible when the CEBAF energy is upgraded to $12 \mathrm{GeV}$.

The method can be easily adapted at lower energies by implementation of the relevant description (phase shift expansion, for instance) of the elementary amplitudes [48]. It may prove useful to predict the physical background and the production cross section of elusive particles, such as pentaquarks.

\section{ACKNOWLEDGMENTS}

I acknowledge the warm hospitality at JLab where this work was completed. The Southern Universities Research Association operates Thomas Jefferson National Accelerator Facility (JLab) for the U.S. Department of Energy under contract no. DE-AC05-84ER40150. Parts of this work were done before I left Saclay.

\section{APPENDIX}

In Ref. [22], the elementary photoproduction amplitude was expressed in terms of Dirac matrices and spinors. In this work, I rewrote it in terms of Pauli matrices and spinors.

For on-shell nucleons, both expressions are equivalent. Both are Lorentz and gauge invariant. They are valid in any frame.

For off-shell nucleons, I made the choice to conserve the three momenta at each vertex and to conserve the energy in the invariant operator but to use the on-shell energy $E=$ $\sqrt{p^{2}+m^{2}}$ in the normalization $\sqrt{E+m}$ and the denominator $E+m$ of Pauli spinors. This choice follows the time-ordered expression of Feynman diagrams [49].

For off-shell nucleons, gauge invariance is lost. However, in the rescattering peaks the target nucleon is almost at rest and the kicked nucleon is on-shell. Therefore the electromagnetic current is conserved in the dominant amplitudes that are considered in this paper.

For the sake of completeness I reproduce the demonstration [50] that I gave about 30 years ago. The quasi-free matrix 
element takes the form

$\mathcal{M}_{\mathrm{I}}=-\bar{u}\left(p_{2}, m_{2}\right) \Theta \frac{\gamma \cdot n+m}{n^{2}-m^{2}} \bar{u}\left(p_{1}, m_{1}\right) \Gamma_{\mu}\left(p_{D}, n\right) \phi_{D}^{\mu}(M)$,

where $\Theta$ is the elementary photoproduction operator, $\Gamma_{\mu}$ is the ${ }^{2} \mathrm{H} n p$ vertex function, and $\phi_{D}^{\mu}$ is the deuteron field.

Retaining only the positive-energy part of the neutron propagator [49] and neglecting its negative-energy part, one gets

$$
\begin{aligned}
\mathcal{M}_{\mathrm{I}}= & -\frac{m}{E_{n}} \sum_{m_{n}} \frac{\bar{u}\left(p_{2}, m_{2}\right) \Theta u\left(\bar{n}, m_{n}\right) \bar{u}\left(\bar{n}, m_{n}\right) \bar{u}\left(p_{1}, m_{1}\right)}{n^{0}-E_{n}} \\
& \times \Gamma_{\mu}\left(p_{D}, n\right) \phi_{D}^{\mu}(M),
\end{aligned}
$$

with $E_{n}=\sqrt{\vec{n}^{2}+m^{2}} \neq n^{0}=E_{D}-E_{1} \quad$ and $\quad \bar{n}=\left(\bar{n}^{0}=\right.$ $\left.E_{n}, \overline{\vec{n}}=\vec{n}\right)$.

Identifying $\Gamma_{\mu}\left(p_{D}, n\right) \phi_{D}^{\mu}(M) m / E_{n}\left(n^{0}-E_{n}\right)$ with the deuteron wave function and defining

$$
T_{\gamma n}\left(\vec{p}_{2}, m_{2},-\vec{p}_{1}, m_{n}\right)=\frac{1}{-i} \bar{u}\left(p_{2}, m_{2}\right) \Theta u\left(\bar{n}, m_{n}\right),
$$

one gets Eq. (2). In terms of Pauli spinors, it takes the form

$$
T_{\gamma n}\left(\vec{p}_{2}, m_{2},-\vec{p}_{1}, m_{n}\right)=\left(m_{2}|\imath \vec{\sigma} \cdot \vec{K}+L| m_{n}\right),
$$

which I use in this work. In the quasi-free amplitude, $\vec{n}=-\vec{p}_{1}$. In the rescattering amplitudes, a similar expression takes into account the actual nucleon momenta.

The vector and scalar parts of the $\pi$ exchange amplitude of the elementary reaction $n\left(\gamma, \pi^{-}\right) p$ are:

$$
\begin{aligned}
& \vec{K}_{\pi}=e g_{\pi N N} \sqrt{2} \frac{\sqrt{\left(E_{n}+m\right)\left(E_{2}+m\right)}}{2 m}\left(t-m_{\pi}^{2}\right) \mathcal{P}_{\text {Regge }}^{\pi^{-}} F_{1}(t) \\
& \times\left[( \frac { \vec { n } } { E _ { n } + m } - \frac { \vec { p } _ { 2 } } { E _ { 2 } + m } ) \left(\frac{\left(2 \vec{p}_{2}-\vec{k}\right) \cdot \vec{\epsilon}}{u-m^{2}}\right.\right. \\
& \left.-\frac{\left(2 \vec{p}_{\pi}-\vec{k}\right) \cdot \vec{\epsilon}}{t-m_{\pi}^{2}}\right)+\frac{\vec{\epsilon}}{u-m^{2}}\left(v-\frac{\vec{p}_{2} \cdot \vec{k}}{E_{2}+m}\right. \\
& \left.-\frac{\vec{n} \cdot \vec{k}}{E_{n}+m}+\frac{v \vec{n} \cdot \vec{p}_{2}}{\left(E_{n}+m\right)\left(E_{2}+m\right)}\right) \\
& +\frac{\vec{k}}{u-m^{2}}\left(\frac{\vec{n} \cdot \vec{\epsilon}}{E_{n}+m}+\frac{\vec{p}_{2} \cdot \vec{\epsilon}}{E_{2}+m}\right) \\
& -\frac{\vec{n}}{u-m^{2}} \frac{v \vec{p}_{2} \cdot \vec{\epsilon}}{\left(E_{n}+m\right)\left(E_{2}+m\right)} \\
& \left.-\frac{\vec{p}_{2}}{u-m^{2}} \frac{\nu \vec{n} \cdot \vec{\epsilon}}{\left(E_{n}+m\right)\left(E_{2}+m\right)}\right] \text {, } \\
& L_{\pi}=e g_{\pi N N} \sqrt{2} \frac{\sqrt{\left(E_{n}+m\right)\left(E_{2}+m\right)}}{2 m}\left(t-m_{\pi}^{2}\right) \mathcal{P}_{\text {Regge }}^{\pi^{-}} F_{1}(t) \\
& \times \frac{1}{u-m^{2}}\left[\frac{\vec{p}_{2} \times \vec{k}}{E_{2}+m}+\frac{\vec{k} \times \vec{n}}{E_{n}+m}\right. \\
& \left.-\frac{v \vec{p}_{2} \times \vec{n}}{\left(E_{n}+m\right)\left(E_{2}+m\right)}\right] \cdot \vec{\epsilon},
\end{aligned}
$$

where $g_{\pi N N}^{2} / 4 \pi=14.5, t=\left(p_{\pi}-k\right)^{2}$, and $u=\left(p_{2}-k\right)^{2}$. I use the Regge propagator $\mathcal{P}_{\text {Regge }}^{\pi^{-}}$that corresponds to the $\pi$ saturating trajectory (as defined in Ref. [22]) and the dipole parametrization of the nucleon isovector form factor:

$$
F_{1}(t)=\frac{4 m^{2}-2.79 t}{\left(4 m^{2}-t\right)(1-t / 0.7)^{2}}
$$

One recognizes the pure $\pi$ exchange amplitude [term in $\left.1 /\left(t-m_{\pi}^{2}\right)\right]$ and the part of the $u$-channel nucleon exchange amplitude [terms in $1 /\left(u-m^{2}\right)$ ] that has been added to ensure gauge invariance (see [22]).

The vector and scalar parts of the $\rho$ exchange amplitude of the elementary reaction $n\left(\gamma, \pi^{-}\right) p$ are

$$
\begin{aligned}
\vec{K}_{\rho}= & e \frac{g_{\rho \pi \gamma}}{m_{\pi}} g_{\rho N N} \sqrt{2} \frac{\sqrt{\left(E_{n}+m\right)\left(E_{2}+m\right)}}{2 m} \mathcal{P}_{\text {Regge }}^{\rho} F_{1}(t) \\
& \times\left(\frac { \vec { p } _ { 2 } \times \vec { n } } { ( E _ { n } + m ) ( E _ { 2 } + m ) } \left\{\left(1+\kappa_{v}\right) V^{0}\right.\right. \\
& \left.+\frac{\kappa_{v}}{2 m}\left[V^{0}\left(n^{0}+E_{2}\right)-\vec{V} \cdot\left(\vec{n}+\vec{p}_{2}\right)\right]\right\} \\
& \left.+\left(1+\kappa_{v}\right) \vec{V} \times\left[\frac{\vec{p}_{2}}{E_{2}+m}-\frac{\vec{n}}{E_{n}+m}\right]\right), \\
L_{\rho}= & e \frac{g_{\rho \pi \gamma}}{m_{\pi}} g_{\rho N N} \sqrt{2} \frac{\sqrt{\left(E_{n}+m\right)\left(E_{2}+m\right)}}{2 m} \mathcal{P}_{\text {Regge }}^{\rho} F_{1}(t) \\
& \times\left(( 1 + \kappa _ { v } ) \left\{V^{0}\left[1+\frac{\vec{n} \cdot \vec{p}_{2}}{\left(E_{n}+m\right)\left(E_{2}+m\right)}\right]\right.\right. \\
& \left.-\vec{V} \cdot\left(\frac{\vec{n}}{E_{n}+m}+\frac{\vec{p}_{2}}{E_{2}+m}\right)\right\} \\
& \left.\times\left[V^{0}\left(n^{0}+E_{2}\right)-\vec{V} \cdot\left(\vec{n}+\vec{p}_{2}\right)\right]\right) . \\
2 m & \kappa_{v}\left[1-\frac{\vec{n}}{\left(E_{n}+m\right)\left(E_{2}+m\right)}\right]
\end{aligned}
$$

I use the Regge propagator $\mathcal{P}_{\text {Regge, with the saturating }}^{\rho}$ trajectory of the $\rho$ meson and the coupling constants $g_{\rho \pi \gamma}=$ $0.103, g_{\rho N N}^{2} / 4 \pi=0.92$, and $\kappa_{v}=6.1$, as in Ref. [22].

The four-vector $V^{\mu}=\left(V^{0}, \vec{V}\right)$ contains the dependency on the polarization vector $\vec{\epsilon}$ of the photon, in the following way:

$$
\begin{aligned}
\left(-k R_{y}, 0, v R_{z}-k R^{0},-v R_{y}\right) & \text { if } \vec{\epsilon}=(1,0,0), \\
\left(k R_{x}, k R^{0}-v R_{z}, 0, v R_{x}\right) & \text { if } \vec{\epsilon}=(0,1,0), \\
\left(0, v R_{y},-v R_{x}, 0\right) & \text { if } \vec{\epsilon}=(0,0,1),
\end{aligned}
$$

where $R^{0}=v-E_{\pi}$ and $\vec{R} \equiv\left(R_{x}, R_{y}, R_{z}\right)=\vec{k}-\vec{p}_{\pi}$ are, respectively, the energy and the three-momentum of the exchanged $\rho$ meson.

For the $p\left(\gamma, K^{+}\right) \Lambda$ reaction, the $K^{*}$ meson amplitudes take the same form as that the $\rho$ meson exchange amplitudes (A8) and (A9), besides trivial changes in the masses, coupling constants, and propagators.

The $K$ exchange amplitude contains the part of the $s$-channel nucleon exchange amplitude that is strictly necessary to ensure 
gauge invariance. It takes the form

$$
\begin{aligned}
\vec{K}_{K}= & e g_{K N \Lambda} \sqrt{2} \frac{\sqrt{\left(E_{n}+m\right)\left(E_{2}+m_{\Lambda}\right)}}{\sqrt{4 m_{\Lambda}}}\left(t-m_{K}^{2}\right) \mathcal{P}_{\text {Regge }}^{K^{+}} \\
& \times\left\{\left(\frac{\vec{n}}{E_{n}+m}-\frac{\vec{p}_{2}}{E_{2}+m_{\Lambda}}\right)\right. \\
& \times\left(\frac{(2 \vec{n}+\vec{k}) \cdot \vec{\epsilon}}{s-m^{2}}+\frac{\left(2 \overrightarrow{p_{K}}-\vec{k}\right) \cdot \vec{\epsilon}}{t-m_{K}^{2}}\right) \\
& +\frac{\vec{\epsilon}}{s-m^{2}}\left[v-\frac{\vec{p}_{2} \cdot \vec{k}}{E_{2}+m_{\Lambda}}-\frac{\vec{n} \cdot \vec{k}}{E_{n}+m}\right. \\
& \left.+\frac{v \vec{n} \cdot \vec{p}_{2}}{\left(E_{n}+m\right)\left(E_{2}+m_{\Lambda}\right)}\right] \\
& +\frac{\vec{k}}{s-m^{2}}\left(\frac{\vec{n} \cdot \vec{\epsilon}}{E_{n}+m}+\frac{\vec{p}_{2} \cdot \vec{\epsilon}}{E_{2}+m_{\Lambda}}\right)
\end{aligned}
$$

$$
\begin{gathered}
-\frac{\vec{n}}{s-m^{2}} \frac{v \vec{p}_{2} \cdot \vec{\epsilon}}{\left(E_{n}+m\right)\left(E_{2}+m_{\Lambda}\right)} \\
\left.-\frac{\vec{p}_{2}}{s-m^{2}} \frac{v \vec{n} \cdot \vec{\epsilon}}{\left(E_{n}+m\right)\left(E_{2}+m_{\Lambda}\right)}\right\}, \\
L_{K}=e g_{K N \Lambda} \sqrt{2} \frac{\sqrt{\left(E_{n}+m\right)\left(E_{2}+m_{\Lambda}\right)}}{\sqrt{4 m m_{\Lambda}}}\left(t-m_{K}^{2}\right) \mathcal{P}_{\text {Regge }}^{K^{+}} \\
\quad \times \frac{1}{s-m^{2}}\left[\frac{\vec{p}_{2} \times \vec{k}}{E_{2}+m_{\Lambda}}+\frac{\vec{k} \times \vec{n}}{E_{n}+m}\right. \\
\left.-\frac{v \vec{p}_{2} \times \vec{n}}{\left(E_{n}+m\right)\left(E_{2}+m_{\Lambda}\right)}\right] \cdot \vec{\epsilon}
\end{gathered}
$$

where $n=\left(E_{n}, \vec{n}\right)$ and $p_{2}=\left(E_{2}, \vec{p}_{2}\right)$ now stand for the four-momentum of the target proton and the outgoing $\Lambda$, respectively, and where $g_{K N \Lambda}^{2} / 4 \pi=10.6$.

As in Ref. [22], here I use the $K$ and $K^{*}$ linear trajectories in the Regge propagators and no hadronic form factor $\left[F_{1}(t)=1\right]$.
[1] J. M. Laget, Phys. Rep. 69, 1 (1981).

[2] G. Bertsch, S. J. Brodsky, A. S. Goldhaber, and J. G. Gunion, Phys. Rev. Lett. 47, 297 (1981).

[3] G. R. Farrar, L. L. Frankfurt, M. I. Strikman, and H. Liu, Phys. Rev. Lett. 64, 2996 (1990).

[4] K. Garrow et al., Phys. Rev. C 66, 044613 (2002).

[5] T. G. O'Neil et al., Phys. Lett. B351, 87 (1995).

[6] An educated guess of the formation length is $l_{f}=2 \hbar c p / \Delta m^{2}$, with $\Delta m^{2} \sim 0.8 \mathrm{GeV}^{2}$. When $p \sim T_{p}=3 \mathrm{GeV}$, one gets $l_{f}=$ $1.5 \mathrm{fm}$.

[7] R. Adams et al., Phys. Rev. Lett. 74, 1525 (1995).

[8] K. Ackerstaff et al., Phys. Rev. Lett. 82, 3025 (1999).

[9] B. Z. Kopeliovich, J. Nemchik, A. Schaefer, and A. V. Tarasov, Phys. Rev. C 65, 035201 (2002).

[10] K. Hadifi, M. Holtrop, and B. Mustapha, spokespersons, JLab Experiment E02-110.

[11] J. M. Laget, in Workshop on JLab Physics at Instrumentation with 6-12 GeV Beams and Beyond, edited by S. Dytman, H. Frenker, and P. Ross, June 1998, p. 57. www.jlab.org/user_ resources/usergroup/workshp.html

[12] L. L. Frankfurt, M. M. Sargsian, and M. I. Strikman, Phys. Rev. C 56, 1124 (1997).

[13] L. L. Frankfurt et al., Phys. Lett. B369, 201 (1996); Z. Phys. A 352, 97 (1995).

[14] W. Boeglin, Eur. Phys. J. A 24, 77 (2005).

[15] K. Egiyan, in International Workshop on Probing Nucleons and Nuclei via the $\left(e, e^{\prime} p\right)$ Reaction, edited by D. Higinbotham, J. M. Laget, and E. Voutier (The Print House, New York, 2004), p. 223.

[16] B. A. Mecking et al., Nucl. Instrum. Methods A 503, 513 (2003).

[17] J. M. Laget, Phys. Lett. B609, 49 (2005).

[18] J. M. Laget, Phys. Rev. D 70, 054023 (2004).

[19] D. Dutta et al., Phys. Rev. C 68, 021001(R) (2003).

[20] H. Gao, R. J. Holt, and V. R. Pandharipande, Phys. Rev. C 54, 2779 (1996)

[21] P. E. Argan, G. Audit, A. Bloch, J. L. Faure, J. M. Laget, J. Martin, G. Tamas, and E. Vincent, Phys. Rev. Lett. 41, 86 (1978).
[22] M. Vanderhaeghen, M. Guidal, and J. M. Laget, Phys. Lett. B400, 6 (1997); Nucl. Phys. A627, 645 (1997); Phys. Rev. C 57, 1454 (1998).

[23] T. Lasinsky et al., Nucl. Phys. B37, 1 (1972).

[24] S. Eidelman et al., Phys. Lett. B572, 1 (2004).

[25] J. M. Laget, in Workshop on color transparency (CT97), edited by E. Voutier (ISN, Grenoble, 1997). http://isnpx0162.in2p3.fr/ polder/ct97/Jlag/Jlag.html

[26] J. M. Laget, in Exclusive and Semi-Exclusive Processes at High Momentum Transfer, edited by C. Carlson and A. Radyushkin (World Scientific, Singapore, 2000), p. 13.

[27] K. Hicks and S. Stepanyan, spokespersons, JLab Experiment E03-113.

[28] M. Lacombe et al., Phys. Lett. B101, 139 (1981).

[29] J. M. Laget, Nucl. Phys. A579, 333 (1994).

[30] J. M. Laget, Nucl. Phys. A296, 388 (1978).

[31] L. Y. Zhu et al., Phys. Rev. C 71, 044603 (2005).

[32] S. Niccolai et al., Phys. Rev. C 70, 064003 (2004).

[33] R. Brun et al., PAW, CERN Program Library Q121.

[34] R. L. Anderson et al., Phys. Rev. D 14, 679 (1976).

[35] M. Battaglieri et al., spokespersons, JLab Experiment E04-017.

[36] P. Nadel-Turonski (private communication).

[37] M. Guidal, Ph.D. thesis, University of Paris, 1996.

[38] J. M. Laget, Phys. Lett. B489, 313 (2000).

[39] F. Cano and J. M. Laget, Phys. Rev. D 65, 074022 (2002).

[40] E. Anciant et al., Phys. Rev. Lett. 85, 4682 (2000).

[41] T. Bauer et al., Rev. Mod. Phys. 50, 261 (1978).

[42] N. N. Nikolaev, A. Szczurek, J. Speth, J. Wambach, B. G. Zakharov, and V. R. Zoller, Nucl. Phys. A567, 781 (1994); Phys. Rev. C 50, 1296 (1994).

[43] L. L. Frankfurt, G. A. Miller, and M. Strikman, Annu. Rev. Nucl. Part. Sci. 45, 501 (1994).

[44] P. Jain, B. Pire, and J. Ralston, Phys. Rep. 271, 67 (1996).

[45] N. Nikolaev, Comments Nucl. Part. Phys. 21, 41 (1992).

[46] T. Nakano et al., Phys. Rev. Lett. 91, 012002 (2003).

[47] S. Stepanyan et al., Phys. Rev. Lett. 91, 252001 (2003).

[48] J. M. Laget, nucl-th/0603009.

[49] F. Gross, Phys. Rev. D 10, 223 (1974).

[50] I. Blomqvist and J. M. Laget, Nucl. Phys. A280, 405 (1977). 